\title{
La Commission des écoles catholiques de Montréal et la prise en compte du pluralisme ethnique et religieux (1977-1998) ${ }^{1}$
}

\author{
Miguel Simao Andrade
}

\section{RÉSUMÉ}

Dans les années 1980 et 1990, l'école franco-catholique, traditionnellement homogène, connait une transformation radicale de sa clientèle sur le plan démographique et doit faire face à une plus grande diversité ethnique qui n'est pas sans entraîner des tensions ethniques. Confrontée à l'afflux considérable des "enfants de la loi 101 ", la Commission des écoles catholiques de Montréal (CECM) se dote d'une politique globale en matière de gestion de la diversité principalement axée sur la valorisation des langues et des cultures d'origine, l'éducation interculturelle et la promotion des relations interethniques. Toutefois, cette adaptation progressive des écoles françaises au pluralisme se heurte à la confessionnalité des structures scolaires et au "projet éducatif chrétien " mis de l'avant par la Commission.

\section{ABSTRACT}

During the 1980's and 1990's, French Catholic schools, homogeneous by tradition, experienced a radical demographical change in clientele and were faced with a greater ethnical diversity that was not without ethnical tension. Confronted with the considerable increase of "Bill 101 children", the Montreal Catholic School Board (MCSB) adopted a global policy regarding the management of diversity based primarily on the appreciation of first language cultures and languages, intercultural education, and on the promotion of interethnic relationships. However, the French schools' gradual adjustment to diversity clashes with the religious nature of their structure and with the "Christian Education Project" developed by the School Board.*

Au Québec, la question de l'intégration des immigrants s'est d'abord posée au niveau des institutions scolaires. Jusqu'en 1998, l'école publique, institution centrale dans le maintien de l'identité ethnique, est confessionnelle et deux réseaux scolaires relativement étanches sont constitués. Le réseau protestant est exclusivement anglophone jusqu'aux années 1960 tandis que le réseau catholique est divisé entre le secteur francophone et le secteur anglophone, créé au milieu du XIX ${ }^{\mathrm{e}}$ siècle pour accueillir les Irlandais catholiques. Dans la première moitié du $\mathrm{XX}^{\mathrm{e}}$ siècle, le repli 
identitaire des francophones empêche l'émergence d'un véritable projet d'intégration et l'écrasante majorité des immigrants choisissent de s'intégrer à la minorité anglophone (protestante et catholique) pour des raisons de mobilité sociale et économique. À la Commission des écoles catholiques de Montréal (CECM), le laissez-faire règne et aucune véritable mesure n'est prise pour favoriser l'intégration des immigrants à l'école francophone. La Commission se préoccupe surtout de l'intégration religieuse et de la préservation de la foi catholique chez les nouveaux arrivants. Au tournant des années 1950, le Comité des Néo-Canadiens de la CECM met en place les premières mesures incitatives visant à favoriser la francisation des immigrants sans toutefois réussir à freiner le mouvement vers l'école anglaise². Dans les années 1960 et 1970, l'émergence de la langue comme critère primordial de l'appartenance nationale et la montée irrésistible du nationalisme québécois transforment profondément les rapports entre la majorité francophone et les minorités ethniques, habituées à s'intégrer à la communauté anglophone ${ }^{3}$. La question de l'intégration à l'école française se retrouve alors au cœur des luttes linguistiques qui secouent la société québécoise. L'État québécois s'affiche comme le représentant de la majorité francophone et assume progressivement ses pouvoirs en matière d'immigration et d'intégration, notamment en adoptant une série de lois restreignant l'accès à l'école anglaise et en élaborant une véritable politique de francisation et d'accueil, notamment par la mise sur pied des premières classes d'accueil en 1969. L'adoption de la loi 101 en 1977, obligeant les immigrants et les minorités ethniques à envoyer leurs enfants à l'école française, clôt la crise linguistique et ouvre un nouveau chapitre dans l'histoire de l'intégration scolaire des immigrants4.

Entre 1977 et 1998, l'école française catholique, historiquement plus homogène que sa consoeur anglaise ou que sa rivale protestante, devient de plus en plus pluraliste. Cette nouvelle réalité soulève la question fondamentale de la gestion des rapports interethniques dans le système scolaire et l'enjeu crucial de la construction d'un espace public commun (l'école) dans un contexte pluriethnique. Pourtant, il est encore difficile d'évaluer les politiques de la CECM en matière d'adaptation au pluralisme pour les années 1980 et 1990 . En effet, aucun historien ne s'est véritablement penché sur cette période plus récente alors que la CECM, confrontée à l'afflux considérable des "enfants de la loi 101 », se dote d'une politique plus globale de gestion de la diversité, principalement axée sur la valorisation des langues et des cultures d'origine, l'éducation interculturelle et la promotion des relations interculturelles et interethniques ${ }^{5}$. En fait, les chercheurs en éducation et en sociologie ont monopolisé les recherches sur les décennies 1980 et 1990 mais la CECM n'a jamais fait l'objet d'une analyse spécifique. Ces derniers ont soit opté pour des études générales sur les rapports entre l'école et les minorités, soit privilégié les analyses comparatives entre les différentes commissions scolaires et entre les systèmes nationaux ${ }^{6}$. Qui plus est, la profondeur diachronique a été partiellement délaissée au profit d'une analyse purement synchronique. L'histoire des politiques de gestion de la diversité ethnique à la CECM dans les années 1980-1990 reste donc à faire.

Plusieurs chercheurs ont laissé entendre que l'école francophone n'était pas prête à subir le « choc multiculturel » de l'après loi 101 et qu'elle était mal outillée pour gérer 
le flot considérable d'enfants immigrants ${ }^{7}$. Si ce constat est vrai, force est d'admettre que la CECM a su s'adapter rapidement à la nouvelle situation. En effet, l'analyse comparative effectuée par Rosalind Zinman sur les politiques interculturelles adoptées par la Commission protestante et par la CECM dans les années 1980-1990 montre clairement que cette dernière était loin d'être en retard sur sa rivale et que les deux institutions défendaient la même vision de l'intégration et du pluralisme ${ }^{8}$. Cependant, des chercheures comme Marie McAndrew et Patricia Lamarre demeurent critiques. Elles considèrent que les mesures prises par les commissions scolaires, protestantes ou catholiques, sont marginales et qu'elles relèvent d'une vision limitée du pluralisme décrite comme du "multiculturalisme additif ». Ces mesures ne modifieraient en rien le fonctionnement général de l'école, en particulier, le curriculum caché, puisqu'elles ne s'appliquent la plupart du temps qu'aux minorités ethniques. Certes, les auteures reconnaissent que l'institution scolaire a cherché à dépasser la simple intégration linguistique et la valorisation des langues et des cultures ethniques en adoptant des mesures concrètes visant à favoriser le rapprochement interculturel. Elles estiment néanmoins que cette évolution demeure incomplète dans la mesure où les écoles francophones montréalaises n'auraient toujours pas réussi à développer chez tous les étudiants un sentiment d'appartenance à la société pluraliste, notamment en raison du fossé séparant les discours, les programmes et les politiques de leur mise en application au niveau local' ${ }^{9}$. Enfin, d'autres chercheurs identifient la confessionnalité scolaire comme le principal obstacle à la reconnaissance du pluralisme ethnique et religieux qui caractérise la société moderne québécoise. La bataille pour la confessionnalité faite dans les années 1970-1980, analysée par Robert Gagnon, met en lumière les tensions nées de la difficile conciliation entre le pluralisme religieux et la confessionnalité scolaire. L'histoire du conflit opposant l'école Notre-Dame-des-Neiges à la CECM sur la question de la déconfessionnalisation telle que décrite par Jocelyne Durand et Jean-Pierre Proulx, en témoigne parfaitement ${ }^{10}$.

Cet article qui s'appuie principalement sur les archives de la $\mathrm{CECM}^{11}$ vise, d'une part, à identifier les principaux enjeux posés par l'émergence du pluralisme ethnique à l'école française dans les années 1980 et 1990 et, d'autre part, à analyser l'adaptation institutionnelle de la CECM en ce qui a trait à l'intégration des minorités ethniques et à la gestion des relations interculturelles. Dans un premier temps, nous chercherons à replacer les politiques scolaires dans le contexte plus général de l'évolution du discours social et des politiques québécoises sur les communautés ethniques. Dans cette optique, les changements aperçus dans les commissions et les écoles seront appréhendés comme le résultat d'un mouvement général qui touche l'ensemble de la société. Dans un deuxième temps, nous dresserons un bilan critique du discours, des mesures et des programmes mis en place par la Commission pour favoriser la reconnaissance et la prise en compte du pluralisme ethnique dans ses écoles. Enfin, nous replacerons cette évolution dans le cadre du débat sur la confessionnalité scolaire afin de poser les limites d'une politique d'ouverture à l'altérité qui s'appuie sur un projet éducatif chrétien, hermétique au pluralisme religieux. Cette analyse permettra de mieux comprendre les paradoxes et les contradictions d'un système scolaire public à la fois commun et confessionnel. 


\section{Un nouveau discours social sur l'altérité}

Durant les années 1970 et 1980, le Québec, tout comme le Canada, assiste à une diversification accrue des flux migratoires internationaux, caractérisée par une forte prépondérance des pays du Tiers-Monde, déchirés par l'instabilité politique et la misère économique. L'arrivée de vagues successives d'immigrants et de réfugiés provenant d'Asie, d'Afrique, d'Amérique latine et des Antilles contraste nettement avec la vieille immigration européenne, plutôt homogène, "blanche " et chrétienne. Entre 1962 et 1984, l'immigration en provenance de l'Europe et des États-Unis chute de $83,9 \%$ à $31,4 \%$ tandis que celle originaire d'Asie fait un bond prodigieux, passant de $7,2 \%$ à $47,5 \%$. Pendant cette période, Haïti se hisse rapidement au premier rang des pays qui fournissent le plus d'immigrants au Québec, suivi du Liban et du Viêt-Nam ${ }^{12}$. Cette diversification des sources d'immigration s'accompagne d'un profond réaménagement des politiques et des idéologies d'intégration et de gestion de la diversité ethnique qui auront un impact considérable sur les politiques scolaires québécoises. Le « virage interculturel » emprunté par la CECM dans les années 1980 et 1990 doit donc être replacé dans le contexte plus général de l'évolution du discours et des politiques québécoises sur les communautés ethniques, caractérisées elles aussi par une plus grande ouverture à l'altérité.

Cette nouvelle dynamique émerge à partir des années 1970 alors que le gouvernement du Québec cherche à devenir le principal pôle d'intégration des communautés ethniques en élaborant sa propre politique de gestion de la diversité, intimement liée à la législation linguistique : l'interculturalisme. Conçue comme une troisième voie, distincte du melting pot américain et du multiculturalisme canadien, la politique québécoise met l'accent sur l'interaction dynamique entre la culture majoritaire et les cultures minoritaires autour d'une langue commune, le français. L'émergence de ce discours axé sur la diversité ethnique et la reconnaissance du rôle des minorités est consacrée par l'adoption de divers énoncés de politiques tels La politique québécoise du développement culturel (1978), Autant de façons d'être Québécois (1981) et le plus récent Au Québec pour bâtir ensemble (1991) ${ }^{13}$. Par ailleurs, l'approche interculturelle s'apparente au multiculturalisme par le fait qu'elle prône le respect et la valeur des cultures d'origine. En effet, dès 1975, la Charte des droits et libertés du Québec enchâsse ce droit en stipulant que « les personnes appartenant à des minorités ethniques ont le droit de maintenir et faire progresser leur propre vie culturelle avec les autres membres de leur groupe ${ }^{14}$.

Dans le domaine scolaire, cette ouverture à l'altérité se traduit concrètement par l'adoption, en 1978, du programme d'enseignement des langues d'origine (PELO) visant à favoriser le maintien des langues et des identités d'origine et à valoriser les apports culturels des communautés immigrantes dans le but, plus général, de faciliter l'intégration scolaire des jeunes allophones et de contribuer au développement d'un sentiment d'appartenance des élèves à l'école française. Entièrement subventionné par le ministère de l'Éducation du Québec (MEQ), le PELO est initialement offert aux élèves des communautés culturelles qui fréquentent les écoles françaises multiethniques. Durant les années 1980, le programme est élargi à l'ensemble des 
élèves, y compris ceux qui fréquentent l'école anglaise. Dans le contexte politique mouvementé de l'après-loi 101, la reconnaissance du pluralisme linguistique au sein du système scolaire envoie un message clair aux immigrants et aux communautés ethniques : l'obligation de fréquenter l'école française n'aura pas comme objectif de leur imposer l'assimilation linguistique, et l'adoption du français comme langue commune n'impliquera pas l'abandon de leur langue d'origine. Certes, dans un premier temps, le PELO suscite la méfiance des minorités ethniques qui le perçoivent à la fois comme une mesure destinée à les amadouer et à rendre la politique de francisation obligatoire plus légitime et comme une menace à la survie de leurs propres institutions scolaires (écoles privés, écoles du Samedi). Cependant, ces résistances initiales se sont progressivement dissipées et l'enseignement des langues d'origine à l'école publique devint une mesure très appréciée des parents immigrants et des leaders ethniques. Dans les années 1990, la clientèle atteint quelque dispersés dans 7000 élèves dispersés dans des classes où l'on étudie 14 langues ${ }^{15}$.

Dans les années 1980 et 1990, le discours québécois sur l'interculturalisme s'est surtout manifesté dans le domaine scolaire, principal lieu de socialisation et d'intégration des communautés ethniques. Le gouvernement québécois reçoit alors une multitude d'avis sur la question de l'école et des minorités, présentés par le Conseil supérieur de l'éducation et la Commission Chancy, chacun insistant sur la nécessaire adaptation des écoles à la réalité pluriethnique du Québec, au moyen de l'adoption d'une politique d'éducation interculturelle fondée sur l'ouverture aux différences culturelles, l'égalité des chances et la lutte contre la discrimination raciale et ethnique $^{16}$. Cette nouvelle approche suppose une remise en cause radicale du fonctionnement, des méthodes, des contenus et des finalités du système scolaire québécois. Le système scolaire, loin d'être culturellement neutre, constitue le principal vecteur de la reproduction culturelle et sociale du groupe majoritaire que ce soit par la transmission d'une langue, de valeurs et d'attitudes communes. Or, pour de nombreux observateurs, la transmission d'une culture publique commune en milieu pluriethnique et l'idéal de l'égalité des chances ne peuvent se réaliser sans la prise en compte de la diversité ethnique.

Sans nier la légitimité de son rôle intégrateur, les partisans de l'éducation interculturelle considèrent donc que l'école québécoise doit également avoir pour mandat de reconnaître l'apport culturel des divers groupes ethniques et d'encourager l'établissement de relations interethniques harmonieuses afin de préparer tous les élèves à vivre ensemble dans une société pluraliste. Pour ce faire, elle doit refléter la diversité de la population qu'elle dessert, et ce, autant dans le contenu des programmes et des manuels qu'au sein de son personnel. À cet égard, l'action du MEQ en faveur d'une meilleure représentation des minorités dans le matériel didactique, notamment par l'élimination des stéréotypes discriminatoires, tout comme son rôle dans la mise sur pied d'un programme de formation et de sensibilisation interculturelle à l'intention du personnel scolaire ont grandement contribué à l'amélioration significative des conditions pédagogiques dans lesquelles s'effectue la transmission des connaissances et des valeurs ${ }^{17}$. Toutefois, la principale recommandation du rapport Chancy concernant l'adoption d'une politique officielle d'éducation interculturelle, maintes fois 
réitérée par divers organismes publics, communautaires et professionnels (Centrale des enseignants du Québec, Conseil de la langue française, Association pour l'éducation interculturelle du Québec), ne sera jamais mise en œuvre par le gouvernement libéral de Robert Bourassa. Invoquant la dimension proprement montréalaise du problème, celui-ci préfere laisser aux commissions scolaires le soin de définir leurs propres politiques en matière d'adaptation au pluralisme ${ }^{18}$. La CECM, principale commission scolaire de Montréal, sera le premier organisme d'éducation à formuler une telle politique pour répondre aux nombreux défis posés par la diversification ethnique de sa clientèle ${ }^{19}$.

\section{Les défis du pluralisme à l'école française}

La Charte de la langue française, adoptée en 1977 au terme d'un long débat sur la langue d'enseignement, transforme radicalement les rapports entre la communauté francophone et les minorités ethniques en révolutionnant le monde scolaire québécois. Concrètement, la loi 101 limite l'accès à l'école anglaise à deux catégories d'enfants : ceux dont le père ou la mère ont reçu l'enseignement primaire au Québec et les frères et les sœurs cadets des enfants qui recevaient déjà l'enseignement en anglais au primaire ou au secondaire dans la province. L'objectif poursuivi est clair : réserver l'école anglaise aux membres de la communauté anglo-québécoise et diriger les enfants des nouveaux immigrants et des minorités ethniques vers l'école française afin de les intégrer à la majorité francophone. Sous les effets conjugués de la loi 101 et d'une immigration de plus en plus diversifiée, largement concentrée dans certains quartiers montréalais, l'école française, autrefois solidement monoethnique, devient pluriethnique. En 1986, les écoles françaises de la CECM comptent 35 \% d'élèves issus des communautés culturelles ce qui contraste nettement avec la situation qui prévalait dans les années 1970 alors que le secteur francophone en comptait un peu moins de $4 \%$. De plus, la présence de ces communautés devient une réalité incontournable pour la plupart d'entre elles : 116 écoles, sur un total de 176, accueillent plus de $20 \%$ d'élèves issus des communautés culturelles, 44 en reçoivent entre 30 et $49 \%$ et 32 comptent une clientèle multiethnique à plus de $50 \%{ }^{20}$.

Par ailleurs, l'arrivée d'une immigration provenant de tous les coins de monde transforme considérablement la composition ethnique des écoles françaises. Aux Italiens et aux Portugais qui arrivèrent nombreux dans les années 1950-1970 se joignent maintenant des immigrants et des réfugiés issus principalement des pays du Tiers-Monde. Ces nouveaux arrivants viennent d'Asie comme les boat people vietnamiens et cambodgiens, des pays d'Amérique latine et des Antilles sous le joug de dictatures autoritaires, comme Haïti et le Chili, ou bien déchirés par de sanglantes guerres civiles, comme le Guatemala et le El Salvador. D'autres viennent du MoyenOrient et d'Afrique. Fait révélateur, au milieu des années 1980, les Haïtiens constituent la principale communauté ethnique à la CECM et la langue créole est la plus parlée, devant l'italien, l'espagnol et le portugais ${ }^{21}$.

Ce changement brusque dans le tissu ethnique des écoles françaises ne se fait pas sans heurts. Défavorisés socialement et économiquement, peu ou pas scolarisés et 
parfois même analphabètes, parlant une langue fort éloigné du français (asiatiques, arabes), plusieurs de ces élèves rencontrent des problèmes d'apprentissage du français et accusent des retards scolaires considérables. Alors qu'en 1979, on observait une intégration scolaire plutôt réussie chez les élèves ayant fréquenté les classes d'accueil, les analyses subséquentes constatent une accentuation des difficultés scolaires due à l'alourdissement de la clientèle et aux coupures budgétaires de 1981 : taux d'abandon plus élevé, retard académique plus important et taux d'échec inquiétant sont présentés comme autant d'entorses au principe fondamental de l'égalité des chances ${ }^{22}$. Ces problèmes d'adaptation scolaire touchent tout particulièrement les élèves haïtiens. Dès le milieu des années 1970, divers groupes de la communauté haïtienne, préoccupés par la situation, organisent des cours de francisation et exercent des pressions auprès du ministère de l'Éducation et des commissions scolaires francophones en vue d'obtenir leur soutien. En 1976, la CECM répond à l'appel en mettant sur pied un Comité spécial chargé d'étudier la situation scolaire des Haïtiens et de proposer les grandes lignes d'un programme qui répondrait aux besoins de cette nouvelle clientèle. Le rapport Ravary, déposé en 1977, révèle que sur les 1800 élèves haïtiens inscrits à la CECM, $56 \%$ accusent un retard scolaire d'un an et $27 \%$ de plus d'un $\mathrm{an}^{23}$. Une enquête faite un an plus tôt fournit d'autres précisions : sur 1013 cas, 499 ont un retard d'un an et 514 de deux ans et plus ${ }^{24}$.

Pour expliquer ces retards scolaires il est primordial de replacer le problème dans le contexte global de l'immigration et de l'intégration des Haïtiens à Montréal. Contrairement à la première vague d'immigration (1968-1972) principalement formée de jeunes professionnels scolarisés fuyant le régime Duvalier, les vagues suivantes sont essentiellement composées de personnes provenant des couches paysanne et populaire, souvent unilingues créolophones ou ne maîtrisant que très peu le français, dont le niveau de scolarité est très faible, sinon inexistant. À Montréal, ces immigrants sont, en outre, confinés aux échelons inférieurs de la hiérarchie sociale et occupent généralement des emplois sous rémunérés. Les difficultés scolaires des jeunes Haïtiens sont donc en lien direct avec les rudes conditions de vie des familles immigrantes : pauvreté, logements inadéquats, sous-scolarisation et déracinement culturel représentent autant de facteurs aggravants ${ }^{25}$. Des causes d'ordre purement scolaire y contribuent également. Par exemple, les responsables pédagogiques ont longtemps refusé d'intégrer les jeunes Haïtiens dans les classes d'accueil parce qu'ils considéraient ces derniers comme des francophones alors que, dans les faits, la très grande majorité parlent le créole. Un rapport du Bureau de l'accueil de la CECM révèle qu'en 1975 seulement 98 élèves haïtiens sur 1 638, soit $6 \%$, fréquentent les classes d'accueil. Il n'est donc pas surprenant que ces élèves aient présenté des difficultés d'apprentissage de la langue française. De plus, le classement académique des élèves en fonction de l'âge chronologique et l'absence d'une analyse systématique du niveau de scolarité des jeunes immigrants auraient repoussé de nombreux Haïtiens vers les classes spéciales ou dans les voies dites allégées ${ }^{26}$.

En 1978, les interventions de la CECM et des associations haïtiennes auprès du MEQ aboutissent à la création d'une "Table de concertation sur les problèmes des enfants haïtiens en milieu scolaire ", réunissant les représentants des ministères et 
des commissions scolaires concernés ainsi que deux organismes communautaires haïtiens. En conclusion du rapport, ces intervenants émettent une série de recommandations visant à favoriser le soutien linguistique et pédagogique des élèves accusant un retard scolaire d'un an et la sensibilisation du personnel scolaire ${ }^{27}$. Il faudra toutefois attendre encore trois ans avant que le MEQ et la CECM s'entendent sur les moyens à prendre pour corriger le problème. Ce long délai ne manque pas de soulever l'exaspération des leaders haïtiens qui prétendent avoir trouvé une oreille plus attentive à la Commission des écoles protestantes du Grand Montréal ${ }^{28}$. En fait, la lenteur du processus s'explique par le fait que le Bureau de l'accueil de la CECM cherche plutôt une solution globale qui pourrait s'appliquer à l'ensemble des communautés ethniques. Il entreprend donc, entre 1980 et 1982, une série d'études sur le problème du retard scolaire chez les immigrants de toutes origines. L'arrivée massive des enfants réfugiés du sud-est asiatique en 1978 et 1979, une clientèle fortement sous scolarisée et analphabète, n'est sûrement pas étrangère à la volonté de la CECM d'élargir le débat aux autres groupes ethniques en difficulté ${ }^{29}$. C'est dans la foulée de ces travaux et grâce à une entente financière avec le gouvernement que la CECM met sur pied, en juin 1982, une nouvelle formule de récupération permettant aux jeunes immigrants, qui ont terminé leur séjour de dix mois en classe d'accueil mais qui accusent toujours un retard scolaire, de poursuivre leur stage dans une classe " post-accueil » pour une période supplémentaire de dix mois. Des mesures particulières sont également prises pour développer des méthodes et du matériel didactique adaptés à la clientèle immigrante et pour favoriser le recrutement d'enseignants " plus sensibles aux valeurs et aux traditions des diverses communautés culturelles $»^{30}$.

Malgré tous ces efforts, les enseignants et les directeurs d'école continuent de déplorer la persistance des retards scolaires chez les jeunes immigrants. Ainsi, l'étude réalisée en 1986 par l'organisme Coopération Nord-Sud en éducation indique que plus de $90 \%$ des intervenants scolaires considèrent que la majorité des élèves néoquébécois qui sortent de l'accueil n'ont pas une connaissance du français suffisante pour suivre le programme régulier ${ }^{31}$. Ce retard occasionne un ralentissement du rythme d'apprentissage dans plus de la moitié des classes de la CECM et un accroissement significatif des tâches de l'enseignant, principalement en ce qui concerne le support linguistique, l'adaptation du programme et, surtout, les relations avec les parents, peu familiers avec les rouages de l'école et les valeurs éducatives québécoises. À cet égard, la sous-représentation des professeurs issus des communautés ethniques dans les écoles françaises tout comme l'absence de représentation des parents néoquébécois au sein de la majorité des comités d'écoles (53\%) ne sont pas sans affecter la qualité des échanges entre les autorités scolaires et les parents ${ }^{32}$. Ce problème de communication s'expliquerait non seulement par l'existence d'une barrière linguistique évidente, mais également par les différences culturelles qui séparent l'école et les familles immigrantes sur la question des valeurs, notamment en ce qui concerne les sévices corporels, le rôle des hommes et des femmes, l'éducation sexuelle et, de façon plus générale, l'opposition que font plusieurs parents immigrants entre l'éducation, chasse gardée de la famille, et l'enseignement, tâche attribuée presque exclusivement au système scolaire ${ }^{33}$. 
Pour les jeunes appartenant aux " minorités visibles " d'immigration récente, l'intégration est d'autant plus difficile qu'elle se déroule parfois dans un contexte où la présence du racisme et de la discrimination entraîne des tensions interethniques qui dégénèrent occasionnellement en violence, aussi bien verbale que physique. À cet égard, le cas des élèves haïtiens, victimes à la fois de difficultés scolaires et de racisme, illustre bien la complexité des nouveaux défis auxquels sont confrontées les écoles françaises, autrefois si homogènes. $\mathrm{Si}$, de façon générale, les relations entre les élèves issus des minorités ethniques et les autres élèves sont jugées harmonieuses, diverses études tendent néanmoins à démontrer que le racisme à l'école est l'une des principales difficultés rencontrées par les minorités visibles, notamment par les élèves noirs. Injures, railleries et blagues racistes proférées par les élèves; hostilité, discrimination ou paternalisme manifestés par des enseignants et des directeurs sont autant de manifestations d'une xénophobie qui, bien qu'elle se vérifie également ailleurs (travail, logement, etc), affecte tout particulièrement les jeunes écoliers en quête de reconnaissance identitaire. Face à la discrimination, ces derniers tendent à se regrouper sur une base ethnique, mais ce mécanisme de défense, communément observé chez tous les groupes, y compris les francophones, est aussitôt mal perçu par certains élèves et enseignants qui voient dans cette pratique un refus de s'intégrer accentuant, par la fait même, les divisions ${ }^{34}$.

À l'aube des années 1980, certaines écoles secondaires de la CECM qui accueillent une nouvelle clientèle haïtienne, comme l'école Henri-Bourassa et l'école LouisJoseph Papineau, font face à une situation particulièrement explosive alors que de violents affrontements interethniques obligent les directions à recourir à des escouades pour rétablir l'ordre entre groupes blancs et noirs. Cherchant à améliorer les relations interethniques, les enseignants de l'école Louis-Joseph Papineau organisent, en avril 1982, la "Semaine des ethnies ", une série d'activités culturelles visant à sensibiliser les jeunes Québécois francophones à la culture des différentes communautés ethniques qu'ils sont appelés à côtoyer quotidiennement ${ }^{35}$. Préoccupée par la montée du racisme dans ses écoles et s'apercevant que les journées de sensibilisation, bien que fructueuses et intéressantes, ne changeraient rien à la situation si elles n'étaient suivies d'actions concrètes, la CECM décide, en juin 1983, de confier à un comité consultatif le mandat d'étudier l'étendue du problème et d'élaborer un plan d'action triennal ayant pour but de prévenir les tensions ethniques ${ }^{36}$. Dès janvier 1984, la CECM accepte de collaborer avec les organismes communautaires haïtiens à la mise sur pied de divers programmes d'animation interculturelle, de rattrapage scolaire et d'alphabétisation à l'intention des élèves haïtiens qui fréquentent les écoles de MontréalNord. De plus, un agent de liaison d'origine haïtienne, Pierre Noël, est engagé pour enquêter spécifiquement sur le problème des tensions ethniques recensé dans trois écoles situées dans cette région et pour œuvrer, plus généralement, à l'amélioration des relations entre l'école et la communauté noire ${ }^{37}$. Sans conclure à un racisme généralisé, le rapport Noël fait néanmoins état de nombreux cas de comportements, de gestes et de propos racistes de la part d'élèves, mais aussi de la part de certains enseignants qui manifestent une xénophobie à tel point que plusieurs de leurs collègues, prenant ouvertement position contre le racisme, considèrent qu'ils devraient changer 
de milieu pour le bien de l'école. Bref, si l'auteur reconnaît que « la majorité des élèves et du personnel enseignant n'ont pas de conduites racistes ", il n'en demeure pas moins qu'une forte proportion d'élèves, soit $78 \%$, se sentent effectivement exclus de leur milieu scolaire, que $64 \%$ des élèves noirs ont le sentiment que certains de leurs enseignants sont racistes et que $56 \%$ disent avoir subi les affronts d'un enseignant ou d'un pair en raison de leur " race ${ }^{38}$. Le rapport, rendu public par la CECM, crée un tapage considérable dans les médias : on parle d'intolérance, de violence et même de risques d'émeutes raciales dans certaines écoles françaises. Se gardant bien d'adopter une attitude aussi alarmiste, la CECM réagit néanmoins avec fermeté en affirmant qu'elle " condamne toute expression de parti pris racial ou ethnique et ne tolère, chez ses élèves et son personnel, sous quelque forme que ce soit, des comportements pouvant provoquer des chocs culturels ${ }^{39}$. Dans les écoles concernées, les directions ne tardent pas à prendre des mesures disciplinaires pour stopper les injures et les comportements racistes chez le personnel scolaire. Intensifiant leur collaboration avec l'agent de liaison de la CECM, ces écoles misent également sur l'organisation d'activités interculturelles et sur le recrutement des parents haïtiens au sein des comités d'écoles pour réduire les tensions raciales. Réagissant à cette même étude, le directeur du Bureau de l'accueil, Robert Attar, soutient que l'ampleur des défis posés par la diversification ethnique des écoles françaises oblige la CECM à se doter d'une politique globale d'éducation interculturelle qui serait non seulement fondée sur l'intégration linguistique des minorités ethniques, mais également sur l'ouverture à la différence et sur le dialogue entre les cultures ${ }^{40}$.

\section{Vers une politique globale en matière de gestion de la diversité ethnique : le « virage interculturel » de la CECM}

Dans les années 1980, l'émergence du concept d'éducation interculturelle aura une influence déterminante sur les politiques de la CECM en matière de gestion de la diversité ethnique. En l'espace de quelques années, le concept gagne du terrain, conquiert de nouveaux adeptes et entraîne une partie importante du monde de l'éducation dans son sillage : avis gouvernementaux, rapports d'organismes publics et communautaires, recherches universitaires, colloques et articles se succèdent alors à un rythme effréné pour tenter de convaincre les décideurs politiques et les administrateurs scolaires d'adhérer à ce nouveau modèle d'intégration des minorités ethniques. C'est justement au milieu de cette décennie, alors que les écoles françaises de la CECM comptent déjà $35 \%$ d'élèves allophones, que le Conseil des commissaires pose les premiers jalons d'une véritable politique d'éducation interculturelle.

L'adoption de la Politique de services aux élèves des communautés culturelles fréquentant les écoles françaises en 1984 constitue la première étape de ce "virage interculturel ». Elle a pour objectif général de "favoriser la prise en charge, par l'école du quartier, d'une éducation interculturelle basée sur la prise de conscience des similitudes entre les ethnies, le respect des différences et l'égalité des chances pour tous les individus ". La politique prévoit la mise en œuvre d'un large éventail de services adaptés aux élèves des communautés ethniques, notamment ceux qui accusent des 
problèmes d'apprentissage linguistique et des retards scolaires importants : conseillers pédagogiques spécialisés, sélection du personnel enseignant en fonction des besoins spécifiques des élèves allophones, formation et perfectionnement des enseignants en éducation interculturelle, production de matériel didactique et d'outils pédagogiques, consolidation des classes post-accueil, multiplication des services d'agent de liaison et d'interprète et mise en place de mécanismes d'information et de sensibilisation auprès des parents. Ces diverses mesurent figurent parmi les principaux moyens pris par la CECM pour relever les défis éducatifs de la pluralité. À l'issue de l'année 1984, les multiples efforts de la CECM sont récompensés par l'obtention du prix national de l'éducation interculturelle du Canada ${ }^{41}$.

En janvier 1985, le MEQ publie le rapport Chancy, intitulé L'École québécoise et les communautés culturelles, véritable plaidoyer en faveur du développement de l'éducation interculturelle où il est proposé à l'école française de s'adapter à la réalité multiethnique du Québec. Ni simple assimilation à la majorité francophone, ni différentialisme ethnique, l'éducation interculturelle implique que " tous les membres de la société, tant ceux qui appartiennent à la culture de la majorité que ceux qui sont membres de groupes ethniques apprennent à respecter tous les groupes et à enrichir leur propre culture au contact des autres ". L'intégration des communautés ethniques à la société québécoise et l'ouverture de la communauté francophone aux apports des cultures minoritaires contribueraient ainsi à l'émergence d'une nouvelle culture québécoise, francophone et pluraliste ${ }^{42}$. S'appuyant sur les recommandations du rapport Chancy, la CECM adopte, en juin 1986, un document intitulé Orientations en regard de l'éducation interculturelle et dont l'objectif est d'amener tous les élèves, peu importe leur origine, à " s'ouvrir à la diversité pour mieux vivre la réalité multiethnique montréalaise ${ }^{43}$.

Dans les années qui suivent, la CECM adopte une série de mesures pour répondre aux multiples exigences de l'éducation interculturelle, notamment dans le domaine de l'enseignement des langues d'origine, des relations avec les communautés culturelles et de la lutte contre la discrimination ethnique. Si l'implantation du Programme d'enseignement des langues d'origine (PELO) à la CECM remonte à 1978, son véritable envol survient au milieu des années 1980 alors que l'ouverture du programme dans les écoles du secteur anglais, en 1982-1983, attire des centaines d'élèves d'origine italienne. Entre 1978 et 1987, le nombre d'élèves inscrits monte en flèche passant de 116 à 2 904. De nouvelles langues font également leur apparition, sous l'impact de l'arrivée massive d'immigrants en provenance des pays d'Asie et d'Amérique latine. Ainsi, les cours d'espagnol, de vietnamien, de laotien, de cambodgien et d'arabe viennent progressivement s'ajouter aux cours de portugais, d'italien et de grec, offerts depuis le tout début ${ }^{44}$.

En 1987, la Commission réitère son engagement en faveur de l'enseignement des langues d'origine en organisant un imposant colloque sur la question et en faisant connaître les résultats positifs de l'évaluation de l'impact du PELO sur les écoles et les élèves. L'étude en question montre que le programme est généralement apprécié des parents et des intervenants scolaires et qu'il a un impact positif sur des aspects comme la sensibilisation interculturelle du milieu scolaire, les liaisons école-communauté, 
l'intégration scolaire des élèves allophones et le développement d'un sentiment d'appartenance à l'école ${ }^{45}$. Toutefois, certains enseignants craignent que l'enseignement de la langue d'origine ne se traduise par une baisse de la performance scolaire et une ghettoïsation des minorités ethniques. D'autres condamnent ouvertement le fait qu'il donne aux élèves allophones la possibilité de devenir trilingues tout en privant les élèves francophones de cet enrichissement culturel ${ }^{46}$. Les observateurs ne s'entendent pas sur le rôle à donner à cet enseignement : doit-il demeurer une mesure spécifique destinée aux seuls allophones dans le but exclusif d'assurer le maintien de leur langue d'origine ou plutôt devenir une mesure d'enrichissement linguistique et interculturel pour tous les groupes? S'appuyant sur une expérience pilote menée par la CECM et sur les revendications d'une majorité d'intervenants, le gouvernement tranche finalement en faveur de la deuxième option et décide, en 1989, d'ouvrir le PELO à l'ensemble de la clientèle scolaire intéressée. Alors qu'il s'inscrivait, depuis sa création, dans une logique de préservation des cultures minoritaires, le PELO évolue alors vers un modèle interculturel ${ }^{47}$. Dans les faits, les francophones ne représenteront jamais plus de $10 \%$ de sa clientèle. Qui plus est, le programme ne jouit pas d'un grand statut ni d'une visibilité importante au sein du système scolaire et sa légitimité est périodiquement remise en question obligeant ses plus chauds partisans à défendre constamment sa valeur pédagogique et sociale. Malgré tout, le PELO continue d'être populaire auprès des communautés ethniques. En 1991, 3555 élèves répartis dans 59 écoles du secteur français et anglais suivent des cours donnés en 9 langues ${ }^{48}$.

Cherchant à intensifier ses relations avec les membres des communautés ethniques et sollicitant leur participation directe et active, la CECM ne se contente plus uniquement d'offrir des services d'agents de liaison et d'interprètes et décide de former, en 1987-1988, un Comité consultatif des groupes ethniques pour qu'il la conseille sur tout ce qui touche les relations avec ces communautés. Des cours de français et d'intégration à la société québécoise sont également offerts aux parents immigrants grâce à la collaboration du ministère des Communautés culturelles et de l'Immigration. En septembre 1988, la CECM met sur pied l'Office des relations interculturelles (ORI) auquel elle confie, outre les activités d'accueil et d'admission et l'application du programme PELO, le développement et la promotion des relations intercommunautaires $^{49}$. Le Service des études de la CECM, quant à lui, s'occupe principalement de la gestion pédagogique du pluralisme en fournissant une panoplie de documents d'orientation et de perfectionnement, de matériel didactique, d'outils d'enseignement et d'évaluation du français en classe régulière ainsi que des guides et des scénarios d'apprentissage spécifiquement destinés à la clientèle sous scolarisée des classes d'accueil et de francisation.

Par ailleurs, durant la deuxième moitié des années 1980, la CECM intensifie ses propres activités de recherche tout en collaborant à plusieurs groupes de recherche ou d'intervention (Centre international d'étude pédagogique, Coopération NordSud en éducation, Conseil de la langue française, Faculté d'éducation de l'université de Sherbrooke, ministère de l'Éducation) dans le but d'évaluer et de perfectionner les services offerts aux élèves allophones. Cet esprit de collaboration se manifeste également dans le domaine de la formation et du perfectionnement en interculturel. 
Soucieuse de préparer l'ensemble de ses intervenants à travailler auprès d'une clientèle pluriethnique, la CECM participe activement à l'élaboration du certificat en éducation interculturelle à l'Université du Québec à Montréal, qui débute en 1987. Grâce à l'appui et à l'expertise du MEQ et du Centre d'éducation interculturelle, la CECM est elle-même en mesure d'offrir à son personnel scolaire (enseignants, cadres, agents de liaison, etc.) une multitude de séminaires, d'ateliers de sensibilisation et de séances de travail qui portent sur des sujets aussi variés que les préjugés et le racisme, les relations entre les écoles et les parents des communautés culturelles, la pédagogie et la communication interculturelle, etc ${ }^{50}$. Enfin, par leurs publications pédagogiques respectives, la CECM et l'Alliance des professeurs de Montréal ${ }^{51}$ contribuent, tout au long des années 1980, à alimenter le débat sur le pluralisme à l'école : articles, dossiers spéciaux et numéros thématiques consacrés à la question de l'intégration scolaire des immigrants constituent non seulement une excellente source d'information sur les programmes existants mais aussi un puissant outil de sensibilisation. À partir de 1990, la publication d'un bulletin de communication interculturelle, Osmose, vient s'ajouter à cet imposant dispositif de communication. Publié par l'ORI à raison de quatre numéros par année et tirés à 11500 exemplaires, Osmose ouvre ses pages aux enseignants et aux professionnels de la CECM actifs sur le terrain des relations interethniques et a comme principal objectif de sensibiliser l'ensemble du personnel scolaire aux questions relatives à l'éducation interculturelle ${ }^{52}$.

En 1988, le cas fort médiatisé de Luis Zuniga, un technicien en informatique d'origine chilienne remercié de ses services par la CECM à cause de son accent trop prononcé, soi-disant incompréhensible, pousse l'ORI et le Comité consultatif des groupes ethniques à se pencher prioritairement sur les problèmes de discrimination et de sous-représentation des minorités ethniques au sein de la Commission. Dès février 1989, ils recommandent l'adoption d'un Plan d'accès à l'égalité en emploi ${ }^{53}$. Un recensement des employés de la CECM confirme l'ampleur du phénomène, surtout dans le secteur français. En effet, si les membres des minorités ethniques représentent $10,45 \%$ des employés réguliers de la CECM, ce pourcentage baisse à $6 \%$ lorsqu'on exclut les enseignants du secteur anglais. Chez les cadres d'écoles du secteur français, la situation est encore pire puisqu'ils ne représentent que 2,67 \% du personnel ${ }^{54}$. La discrimination directe et systémique tout comme l'arrivée relativement récente de la population immigrante dans le système scolaire francophone sont, en grande partie, responsables de cette nette sous-représentation, sans compter l'impact de la longue période de décroissance de l'effectif scolaire qui n’a certainement pas favorisé l'embauche de personnel nouveau ${ }^{55}$.

Prenant la parole à l'occasion d'une vaste consultation sur les relations entre l'école et les communautés culturelles réunissant, en avril 1989, plus de 300 participants, le président de la CECM, Michel Palascio, s'engage publiquement à mettre en ouvre une politique d'accès à l'égalité visant à assurer une représentation adéquate des minorités aux différents niveaux de l'organisation et à supprimer les règles et les pratiques du système d'emploi (recrutement, affectation, promotion) susceptibles d'être discriminatoires. Aussi, profite-t-il de l'occasion pour dévoiler la Déclaration de la CECM contre la discrimination raciale et le racisme qui fait suite aux déclarations 
similaires adoptées par le gouvernement du Québec et la Ville de Montréal, en 1986 et 1989 respectivement. S’appuyant sur la Charte des droits et libertés de la personne du Québec, les autorités de la CECM « condamnent et refusent de tolérer toutes formes de non-respect, ayant une connotation de discrimination raciale ou ethnique de la part de ses commissaires gestionnaires membres du personnel ou élèves » et " s'engagent à favoriser dans les écoles une éducation exempte de stéréotypes et de préjugés raciaux ${ }^{56}$.

Par ailleurs, ce discours officiel d'ouverture au pluralisme masque parfois des résistances larvées et des craintes vis-à-vis le phénomène de la concentration ethnique. Par exemple, à l'automne 1989, la Commission souhaite effectuer un sondage auprès des parents pour savoir s'ils sont en faveur de l'établissement d'écoles séparés pour les minorités ethniques. Si le tollé médiatique suscité par la question oblige finalement la CECM à se rétracter, il n'en reste pas moins que le simple fait d'avoir envisagé la possibilité d'établir une ségrégation ethnique entre les écoles indique bien l'ambivalence des commissaires à l'égard de la diversité ethnique. À la même époque, le projet de la CEPGM, principale commission scolaire protestante de Montréal, visant à concentrer ses élèves d'origine haïtienne dans certaines écoles est abandonné après avoir suscité le même type de protestations ${ }^{57}$. En 1990, le Regroupement scolaire confessionnel (RSC), sous le leadership du président Palascio, se prononce en faveur d'une politique d'immigration qui privilégie la sélection d'immigrants francophones de tradition judéo-chrétienne, jugés plus aptes à s'intégrer harmonieusement à la société québécoise. Cette recommandation, adoptée à quelques jours des élections scolaires, soulève l'indignation et les critiques acerbes chez les représentants des communautés ethniques et religieuses, mais aussi l'irritation de plusieurs ministres. Le président du Mouvement pour une école moderne et ouverte (MEMO), principal parti d'opposition à la CECM, qualifie la position du RSC de " raciste " et d' "intégriste " tandis que la présidente du Centre maghrébin de recherche et d'information déplore le conservatisme rétrograde du président de la CECM, un homme qui, selon elle, " tient un discours d'exclusion plutôt que d'intégration des communautés religieuses et d'ouverture ». De son côté, le président de l'Alliance, Denis Gendron, tient à dissocier les 8000 professeurs de la CECM qu'il représente, en affirmant qu'une telle position " détruit les efforts d'intégration des communautés et d'harmonisation que nous poursuivons dans les écoles $"^{58}$.

Si ces dérapages politiques sont effectivement nuisibles aux efforts pour rapprocher la CECM des communautés culturelles, ils ne remettent pas nécessairement en cause l'adaptation institutionnelle au pluralisme, déjà bien enclenchée. En avril 1990, l'Office des relations interculturelles dépose, en effet, un plan d'action intitulé $L e$ développement et la promotion des relations interculturelles et interethniques à la CECM qui se veut la concrétisation des orientations en éducation interculturelle adoptées en 1986. Visant à rendre " le personnel, les élèves jeunes et adultes ainsi que les parents plus aptes à vivre dans une société de langue française et pluraliste ", ce plan s'appuie sur les concepts de société pluraliste, d'éducation interculturelle, d'interactions dynamiques, de prévention et de lutte contre la discrimination, de francisation et d'égalité des chances. Les efforts consacrés par l'ORI au règlement de cette dernière question 
aboutissent finalement, en 1992, par l'adoption officielle d'un Programme d'accès à l'égalité en emploi pour les communautés culturelles et d'une Politique concernant les moyens de contrer le harcèlement racial qui prévoit la création d'un comité de recours où des plaintes formelles pourront être traitées ${ }^{59}$.

Durant les années 1990, plusieurs études viennent signaler les dérives potentielles d'une vision relativiste de l'éducation interculturelle trop centrée sur les différences et les particularismes ethniques : réification et folklorisation des identités culturelles, renforcement des frontières ethniques, risque d'ethnocentrisme et d'intolérance, imposition d'une identité figée, affaiblissement de la cohésion sociale, etc. Ce courant intellectuel affirme l'importance de concilier la diversité avec la cohésion sociale par l'adhésion à une langue et à des valeurs communes à tous les citoyens québécois ${ }^{60}$. Sous les effets de ces critiques, l'éducation interculturelle à la CECM s'oriente de plus en plus vers une perspective civique qui mise moins sur la valorisation des différences culturelles que sur les ressemblances et l'affirmation de valeurs communes. Ainsi, les activités pédagogiques et les "semaines des communautés ethniques " qui consistaient, bien souvent, en une simple juxtaposition de kiosques folkloriques, deviennent plutôt des lieux d'échange et de réflexion sur des thèmes comme celui de «culture publique commune » ou de " contrat moral » d'intégration définissant ce qui est négociable et ce qui ne l'est pas dans la société québécoise. Le bilan des activités interculturelles, réalisées dans les écoles de la CECM entre 1990 à 1995, laisse déjà percevoir ce changement d'orientation ${ }^{61}$.

\section{La CECM et la reconnaissance du pluralisme religieux à l'école}

Si la CECM adopte, dans les années 1980 et 1990, un discours et des politiques favorisant la reconnaissance et la prise en compte du pluralisme ethnique, sa défense de la confessionnalité scolaire et de l'éducation catholique constitue toutefois un obstacle majeur à l'intégration des immigrants non catholiques à l'école française et à la pleine reconnaissance de la diversité religieuse ${ }^{62}$. Depuis le début du XX $X^{e}$ siècle, la quasi-totalité des immigrants non catholiques optent pour l'école protestante tandis que la CECM, fortement homogène au point de vue religieux, se préoccupe surtout de l'éducation des catholiques. Encore au milieu des années 1960, le nombre d'élèves " neutres " à la CECM n'est que de 203 tandis que la Commission protestante en accueille près de 4000 , sans compter les milliers d'enfants juifs ${ }^{63}$. L'évolution de la société québécoise dans les années 1960, marquée par le mouvement de laïcisation, la désaffection religieuse et par l'arrivée de nouveaux groupes n'appartenant pas aux confessions chrétiennes, remet en cause la pertinence de cette définition séculaire de l'éducation. Dès cette époque, plusieurs voix se lèvent pour réclamer un assouplissement de la confessionnalité afin que l'école puisse respecter le pluralisme religieux de la société québécoise ${ }^{64}$.

Mais contrairement à la Commission protestante, historiquement plus neutre au niveau confessionnel, la CECM tarde à s'adapter aux réalités du pluralisme religieux ${ }^{65}$. Dans les années 1970, cette dernière applique encore difficilement le droit à l'exemption des cours d'enseignement religieux. Plutôt que d'adopter une ligne 
directrice et cohérente, la Commission laisse aux directions scolaires le soin d'informer les parents sur leurs droits. Par conséquent, le respect de la liberté religieuse varie considérablement d'un établissement à l'autre en fonction des convictions religieuses du milieu scolaire et des pressions extérieures, souvent exercées par l'Association des parents catholiques. Au milieu des années 1970, un rapport sur le sujet constate que l'information donnée aux parents «va du mutisme le plus complet au prosylétisme à peine voilé " et que les cours de morale pour les élèves exemptés de cours de religion sont rarement offerts ${ }^{66}$. À l'extérieur de la Commission, un groupe de parents, soucieux d'assurer le respect de la liberté de conscience, met sur pied, en mars 1976, l'Association québécoise pour l'application du droit à l'exemption de l'enseignement religieux. Les pressions exercées par cet organisme militant poussent la CECM à apporter certaines améliorations, mais il faut toutefois attendre 1985 avant que le régime d'exemption ne soit définitivement remplacé par un régime d'option entre l'enseignement religieux et l'enseignement moral offrant à tous les élèves un véritable choix ${ }^{67}$.

Si la CECM accepte timidement de faire certains accommodements au niveau de l'enseignement religieux, sa position concernant la confessionnalité scolaire ne tolère, par contre, aucun type de compromis. L'écrasante victoire électorale des commissaires affiliés au Mouvement scolaire confessionnel (MSC), en 1977, n'a fait que radicaliser sa position en cette matière ${ }^{68}$. Les commissaires du MSC, groupe conservateur ultra-catholique, devenu le Rassemblement scolaire confessionnel (RSC) en 1990, gardent les rênes de la plus grande commission scolaire du Québec pendant une vingtaine d'années à une époque où, paradoxalement, les valeurs religieuses traditionnelles perdent du terrain au profit d'une vision moderne, pluraliste et laïque de la société québécoise. Défenseurs de la confessionnalité scolaire pour les catholiques et ardents promoteurs des " écoles catholiques où les valeurs chrétiennes doivent être présentées dans tout le programme et non seulement dans les cours de religion ", ces commissaires chercheront par tous les moyens à modeler la CECM à l'image de leur philosophie religieuse. Dès 1977 , le "projet éducatif chrétien " visant à transmettre une éducation chrétienne inspirée de l'Évangile et de l'Église se retrouve au cœur des grandes orientations de la $\mathrm{CECM}^{69}$. Même les élèves fréquentant les classes d'accueil n'échappent pas à cette vaste opération de réaffirmation et d'approfondissement des valeurs catholiques. En effet, si la diversité religieuse au sein de ces classes avait, jusque-là, empêché l'établissement d'un programme d'enseignement religieux, ce n'est plus le cas sous le règne du nouveau Conseil qui s'empresse d'ordonner le mise en oeuvre des « éléments de base permettant aux professeurs des classes d'accueil d'assurer l'éveil religieux de leurs élèves et leur préparation aux principaux événements de la liturgie chrétienne ${ }^{70}$.

Dans le tournant des années 1980, la bataille pour la confessionnalité reprend de plus belle alors que les commissaires, majoritairement favorables au maintien strict de la confessionnalité, sont confrontés au comité de parents de l'école multiethnique Notre-Dame-des-Neiges (NDDN) qui réclame un statut non-confessionnel et l'adoption d'un projet d'éducation pluraliste ouvert à tous les élèves du quartier. À la fin des années 1970, l'école NDDN accueille près de $30 \%$ d'élèves dont la langue 
maternelle et la confession religieuse ne sont pas celles de la majorité canadiennefrançaise catholique. Cette diversification ethnoreligieuse se traduit notamment par une hausse constante du pourcentage d'exemptés qui passe de $26 \%$ en 1975 à 36 $\%$ en $1978^{71}$. La confessionnalité scolaire en milieu pluriethnique apparaît de plus en plus clairement, aux yeux de plusieurs autorités scolaires et politiques, comme un obstacle à l'intégration des élèves non-catholiques et à la reconnaissance de la diversité religieuse. S'appuyant sur un sondage mené auprès des parents qui démontre que 90 \% d'entre eux souhaitent que l'école devienne pluraliste, le comité d'école propose, en février 1979, que la CECM demande au comité catholique du MEQ de révoquer le caractère confessionnel de l'école NDDN afin que cette dernière puisse refléter la réalité multiculturelle du quartier Côte-des-Neiges ${ }^{72}$.

Mais en dépit de la volonté affirmée d'une majorité de parents, de la décision favorable et unanime du comité catholique, de l'accord du ministre de l'Éducation et de l'appui de nombreux organismes (CEQ, Alliance des professeurs, Conseil central des parents), le Conseil des commissaires de la CECM refuse cette requête. Déterminé à " protéger les droits à l'éducation catholique de la très grande majorité de la population ", la CECM donne également son appui à cinq parents catholiques qui contestent devant les tribunaux la juridiction du comité catholique en matière de reconnaissance et de révocation du statut confessionnel des écoles ${ }^{73}$. Cette première bataille judiciaire vise surtout à faire déclarer que " toutes les écoles assujetties à la juridiction de la CECM, et, en particulier, l'école NDDN, sont de plein droit des écoles confessionnelles " en vertu de l'article 93 de l'Acte de l'Amérique du Nord britannique $^{74}$. Elle se solde par une victoire des tenants de la confessionnalité lorsque la Cour supérieure du Québec donne raison à la CECM. Dans son jugement, le juge Deschênes confirme que les écoles de Montréal et de Québec sont confessionnelles et annule, par le fait même, la résolution du comité catholique quant à la révocation du caractère catholique de l'école NDDN.

Ce jugement porte un dur coup aux partisans de l'école confessionnelle qui sont pourtant devenus largement majoritaires si on se fie à l'appui des multiples organisations québécoises. En effet, en 1983, les deux grands partis politiques québécois - le Parti Québécois et le Parti libéral du Québec — les centrales syndicales, la Fédération québécoise des comités de parents, la Fédération des commissions scolaires catholiques et même l'Assemblée des évêques du Québec se sont tous ralliés à l'avis émis par le Conseil supérieur de l'éducation concernant l'établissement de commissions scolaires linguistiques. Or, tout au long des années 1980, les multiples tentatives de réformes législatives visant à déconfessionnaliser le système scolaire québécois et à le refondre sur une base linguistique vont, à chaque fois, se buter soit aux contraintes constitutionnelles et aux décisions défavorables des tribunaux, soit à l'opposition farouche des regroupements confessionnels radicaux et, en particulier, de la $\mathrm{CECM}^{75}$.

Contre les tenants de la laïcité qui invoquent les principes de la neutralité de l'Etat et des droits individuels, les partisans de l'école confessionnelle défendent l'idée de droits collectifs, de tradition, d'histoire. Ils font valoir que l'enseignement religieux contribue à la transmission des valeurs judéo-chrétiennes sous-jacentes à la culture d'une majorité de Québécois. Sous la présidence de Michel Palascio, la CECM 
réaffirme encore plus fermement sa volonté d'imposer à toutes ses écoles un projet éducatif "basé sur une proposition explicite de la foi chrétienne ». En 1985, le Plan de promotion des valeurs chrétiennes, adopté par le Conseil des commissaires, encourage, par exemple, "l'expression et l'habitude de la prière personnelle et collective " et suggère l'ajout d'images religieuses, de crucifix et de signes visuels de la foi dans la décoration des écoles ${ }^{76}$. Ce durcissement contribue à susciter l'émergence d'un parti d'opposition, le Mouvement pour une école moderne et ouverte (MEMO), qui se prononce en faveur des commissions scolaires linguistiques, mais, faute d'appuis électoraux, ce dernier ne réussira jamais à infléchir la politique de la CECM en ce sens.

Par ailleurs, l'accentuation du caractère rigide et monolithique de l'école francocatholique ne peut qu'avantager l'école franco-protestante, plus neutre en matière religieuse. Cette dernière connaît d'ailleurs une expansion sans précédent dans les années 1980 et continue d'attirer la très grande majorité des élèves professant d'autres confessions. Par exemple, en 1993-1994, 66 \% de sa clientèle n'est pas protestante. La CECM, quant à elle, compte $20 \%$ d'élèves non catholiques, dont 3,9\% de musulmans, $3 \%$ de bouddhistes, $2 \%$ d'orthodoxes, 1,9 \% de protestants, 1,9\% d'hindouistes, $0,6 \%$ de témoins de Jéovah et 7,3\% qui déclarent n'avoir aucune religion ${ }^{77}$. Il ne faut toutefois pas sous-estimer le pluralisme croissant des écoles franco-catholiques d'autant plus que les statistiques globales négligent le phénomène de concentration religieuse dans certaines écoles. Ce nouveau pluralisme engendre des situations particulièrement délicates comme le précise un observateur averti dans un article intitulé "S'accommoder n'est pas commode " publié dans la revue interculturelle de la CECM, Osmose :

Une élève de neuf ans jeûne pendant le mois de Ramadan; des adolescents, Témoins de Jéhovah, s'absentent d'une école secondaire pour participer à une quinzaine de prières; de jeunes musulmans réclament un lieu pour prier le midi, d'autres insistent pour être exemptés du cours d'éducation physique parce qu'il est mixte ou du cours de musique jugé trop profane. Si l'on ajoute, à ces exemples, les demandes déjà connues touchant le port du voile islamique en classe pour les musulmans, celui de la casquette pour les Noirs et du poignard rituel pour les Sikhs, on commence à avoir une idée des situations délicates que le pluralisme engendre dès à présent dans nos écoles ${ }^{78}$.

Dans les années 1990, le concept juridique « d'accommodement raisonnable », apparu au milieu des années 1980 comme une mesure destinée à protéger les droits individuels fondamentaux en cas de discrimination ${ }^{79}$, acquiert un sens plus large lié à la notion d'aménagement négociée au pluralisme. Pour la plupart des intervenants scolaires, peu férus en droit constitutionnel, les accommodements désignent, dans leur pratique quotidienne, la formule de compromis par laquelle une institution accepte de s'adapter à certaines normes culturelles et religieuses, dans les limites compatibles avec les règles civiques communes. Cette négociation culturelle apparaît de plus en plus comme le principal outil de gestion du pluralisme culturel et religieux dans les écoles montréalaises. Mais, comme il n'existe aucune balise officielle, ni du côté gouvernemental, 
ni de la part des commissions scolaires, chaque école est obligée d'improviser un code d'éthique de sorte que les pratiques concernant les accommodements religieux dépendent souvent du bon vouloir des directions d'école et des enseignants et peuvent donc varier considérablement d'un établissement scolaire à l'autre ${ }^{80}$.

Par exemple, en 1992, l'école La Dauversière, fréquentée par une forte population orthodoxe, décide de tenir compte de son calendrier religieux en offrant une journée de congé pascal pour les élèves de cette confession alors que, jusqu'à cette date, certains enseignants plaçaient volontairement un examen lors des fêtes religieuses orthodoxes pour obliger les élèves à suivre le calendrier catholique ${ }^{81}$. Au contraire, d'autres écoles refusent catégoriquement de s'adapter au pluralisme religieux et de consentir la moindre concession aux membres des minorités religieuses comme en témoigne la "crise du hijab » de 1994-1995 à la CECM. La décision prise par l'école LouisRiel, en 1994, d'expulser une fillette de 13 ans parce qu'elle désirait porter le voile islamique déclenche un débat passionné et complexe qui aboutit, un an plus tard, à la publication d'un avis de la Commission des droits de la personne établissant que les écoles publiques sont tenus de respecter la liberté religieuse garantie par la Charte et n'ont, par conséquent, pas le droit d'interdire l'accès à des élèves voilées ${ }^{82}$.

Durant les années 1990, les têtes dirigeantes de la CECM n'interviennent pas directement dans ce débat épineux et aucune politique administrative n'est adoptée pour fixer des balises claires aux accommodements raisonnables. Par conséquent, cette délicate tâche incombe exclusivement aux acteurs locaux sur le terrain scolaire soit les directeurs d'école, les parents concernés et le conseiller en relations interculturelles de la CECM qui joue un rôle clé de médiation. Des dizaines de crises potentielles ont ainsi pu être évitées grâce à des accords conclus à l'issue de négociations se déroulant dans l'ombre, loin des projecteurs médiatiques ou des salles des tribunaux ${ }^{83}$.

Le débat sur la gestion du pluralisme religieux en contexte scolaire n'est évidemment pas spécifique à l'école confessionnelle québécoise et il suffit de se référer aux difficultés vécues par le modèle de l'école laïque en France pour s'en convaincre. Il n'en demeure pas moins que la déconfessionnalisation du système scolaire et l'établissement de commissions scolaires linguistiques, en 1998, lèvent un des principaux obstacles à la reconnaissance du pluralisme religieux. Cette même année, le MEMO goûte pour la première fois au pouvoir mettant ainsi fin au long règne du Regroupement scolaire confessionnel. Ces ruptures d'ordre structurel et politique annoncent une nouvelle ère pour la nouvelle Commission scolaire de Montréal (CSDM). Par ailleurs, la déconfessionnalisation scolaire ne règle pas pour autant le débat beaucoup plus large sur la place de la religion à l'école publique, bien au contraire. Le rapport Proulx l'avait déjà noté en $1999^{84}$. La récente controverse concernant la question des accommodements raisonnables le confirme : le grand défi actuel du système scolaire québécois est, sans aucun doute, celui de l'aménagement du pluralisme religieux dans une société laïque ${ }^{85}$.

Dans ce contexte brûlant d'actualité, la Commission scolaire de Montréal se retrouve encore une fois au cœur du débat. Comptant aujourd'hui près de $50 \%$ d'élèves allophones provenant de 195 pays, parlant 150 langues et pratiquant diverses religions, dont le bouddhisme, le catholicisme, le protestantisme, l'hindouisme, les 
religions juive, orthodoxe et sikhe, la CSDM représente un véritable laboratoire de la multiethnicité montréalaise. En 2006, elle adopte une ambitieuse politique interculturelle visant à concilier les principes de laïcité, de pluralisme, d'intégration et d'identité commune. Pour une première fois dans l'histoire de l'éducation au Québec, les contours d'une laïcité ouverte et respectueuse des choix individuels sont fixés par des balises régissant ce qui est acceptable ou non-acceptable dans les cas d'accommodements raisonnables ${ }^{86}$. L'année suivante, en pleine tempête médiatico-politique, le gouvernement libéral dirigé par le premier ministre Jean Charest met sur sur pied un Comité consultatif sur " l'intégration et l'accommodement raisonnable en milieu scolaire ». Regroupant divers acteurs du monde scolaire, le Comité est présidé par nul autre que Bergman Fleury, l'expérimenté conseiller en éducation interculturelle de la CSDM. Le rapport Fleury qui s'inspire notamment des efforts entrepris depuis plus de 10 ans à la CSDM est sûrement appelé à devenir la référence pour toutes les écoles québécoises ${ }^{87}$.

\section{Conclusion}

Entre 1977 et 1998, l'école franco-catholique connaît une diversification ethnique croissante de sa clientèle, sous l'impact conjugué de la loi 101 et d'une immigration de plus en plus diversifiée. L'irruption soudaine du pluralisme dans un milieu traditionnellement homogène ne se fait pas sans heurts. Les problèmes d'apprentissage linguistique et les retards scolaires touchent particulièrement les immigrants sous-scolarisés issus des pays en voie de développement. Les tensions interethniques et le racisme apparaissent également au grand jour, particulièrement dans les écoles fréquentées par les minorités visibles. L'étude du cas particulier des élèves haïtiens, victimes à la fois de retards scolaires et de racisme, nous a permis d'illustrer la complexité des enjeux liés à l'adaptation institutionnelle au pluralisme. Influencée par l'émergence d'un nouveau discours social sur l'altérité fondé sur l'interculturalisme, la CECM se dote, au milieu des années 1980, d'une politique globale de gestion du pluralisme principalement axée sur la valorisation des langues d'origine, l'éducation interculturelle et la promotion des relations interethniques. Le processus d'intégration, distinct de l'assimilation, est défini comme le résultat des interactions entre la majorité et les minorités autour d'une langue commune, le français, dans le respect des différences culturelles. Un large éventail de mesures et de programmes sont mis sur pied pour entreprendre ce virage interculturel visant à adapter l'école à la réalité pluriethique montréalaise. Programme d'enseignement des langues d'origine, classes post-accueil, programmes de formation et de perfectionnement du personnel scolaire, matériel didactique et outils pédagogiques, activités interculturelles, agents de liaison, conseil des communautés culturelles, plan d'accès à l'égalité et de lutte contre la discrimination ethnique témoignent bien de l'ampleur du travail accompli dans les années 1980 et 1990 .

Malgré cette adaptation réussie au pluralisme ethnique, la confessionnalité scolaire et le projet éducatif chrétien mis de l'avant par la CECM constituent des obstacles majeurs à l'intégration des immigrants non-catholiques et à la pleine reconnaissance 
du pluralisme religieux. La CECM n’a donc jamais pu négocier complètement son virage interculturel. Cette dernière demeure toute au long de son histoire une institution catholique qui s'intéresse surtout à l'intégration des immigrants catholiques. Si la confessionnalité scolaire constitue un obstacle légal à la reconnaissance du pluralisme religieux, elle ne suffit pas à expliquer cette indifférence à l'égard des non-catholiques. Après tout, la Commission protestante, soumise aux mêmes réalités structurelles, s'est montrée plus ouverte aux immigrants d'autres confessions religieuses, notamment en offrant un enseignement plus neutre au niveau confessionnel. Certes, l'existence de différentes branches au sein même du protestantisme prédispose la CEPGM à une plus grande tolérance à l'égard de la diversité des croyances alors que la CECM, héritère d'une tradition religieuse fondée sur l'universalité et l'uniformité de la doctrine, semble a priori plus hermétique au pluralisme religieux. Toutefois, celle-ci aurait très bien pu, malgré le poids de son héritage catholique, favoriser davantage l'intégration des élèves non-catholiques, notamment en reconnaissant plus facilement certains droits comme l'exemption des cours d'enseignement religieux ou la déconfessionnalisation de certaines écoles multiethniques. Pourquoi ne l'a-t-elle pas fait? D'abord, leur faible nombre l'a confortée pendant de nombreuses années sur le bien-fondé de sa politique. A ses yeux, il n'incombait pas à l'institution scolaire de se conformer à l'infime minorité de non-catholiques, mais plutôt à celle-ci de se conformer aux intérêts de l'écrasante majorité catholique. Ensuite, lorsque le nombre de non-catholiques commence à augmenter dans les années 1980, la CECM est dirigée par un groupe de commissaires conservateurs et traditionnels voués au maintien strict de la confessionnalité scolaire et à la défense d'un projet éducatif chrétien. Les rapports conflictuels entre la CECM et l'école Notre-Dame-des-Neiges illustrent bien les paradoxes et les contradictions d'un système scolaire à la fois commun et confessionnel.

Par ailleurs, nous avons vu que les rapports entre les minorités et l'école franco-catholique évoluent rapidement au cours des années 1990 alors que plusieurs directions scolaires acceptent de négocier des accommodements raisonnables dans le but de favoriser l'intégration des minorités religieuses dans le respect des droits individuels. L'existence même de cette adaptation négociée au pluralisme met en lumière l'importante autonomie des acteurs scolaires sur le terrain local et les limites du pouvoir central incarné par le Conseil des commissaires. Certaines écoles multiethniques ont ainsi pu contribuer à l'aménagement d'un espace de liberté religieuse et d'égalité des droits malgré l'existence d'une structure confessionnelle assez stricte. Mais la véritable rupture survient en 1998 avec la mise en place des commissions scolaires linguistiques qui met finalement un terme à la division confessionnelle du système scolaire et ouvre ainsi la voie à l'émergence d'un véritable espace scolaire commun. À l'aube du XXI siècle, les écoles publiques françaises, contrôlées par la majorité francophone, deviennent finalement l'unique lieu d'accueil et d'intégration des communautés immigrantes et ethniques, peu importe leur appartenance ethnique ou religieuse. La récente contribution de la Commission scolaire de Montréal dans le débat sur la laïcité, le pluralisme religieux et les accommodements raisonnables confirme cet engagement dans la construction d'un espace public commun ouvert au pluralisme. 


\section{Notes}

* La traduction du résumé est d'Alexandre Dussault et de Catherine Élie, sous la supervision de leur professeure d'anglais, Diane Pigeon.

1 Cet article est tiré de mon mémoire de maîtrise. Voir Miguel Simao Andrade, La CECM et l'intégration des immigrants et des communautés ethniques, 1947-1998, mémoire de maitrise (histoire), UQAM, 2005. J'aimerais remercier mon directeur Robert Gagnon et Paul-André Linteau dont les précieux commentaires ont contribué à améliorer cet article.

2 Michael Behiels, "The Commission des Écoles catholiques de Montréal and the Neo-Canadian Question : 1947-1963 ", Canadian Ethnic Studies 18, 2 (1986) : 38-64; Robert Gagnon, "Pour en finir avec un mythe : le refus des écoles catholiques d'accepter les immigrants ", Bulletin d'histoire politique 5, 1 (hiver 1997) : 120-141; Miguel Simao Andrade, "La CECM et l'intégration des minorités ethniques : de la foi à la langue ", in Jean-Michel Lacroix et Paul-André Linteau, dir., Vers la construction d'une citoyenneté canadienne (Paris : Presses de la Sorbonne Nouvelle, 2006), 49-76.

3 Dans cet article, les termes de communautés et de minorités ethniques désignent l'ensemble des individus regroupant les immigrants et leur descendance qui appartiennent à une communauté, distincte des Canadiens français et des Canadiens anglais par leur statut minoritaire. Les groupes ethniques sont définis en fonction du processus qui renvoie à la construction sociale de l'ethnicité, soit le rapport inégalitaire entre la majorité et les minorités, constitutifs des " frontières ethniques ». Pour une analyse sociologique de l'ethnicité, voir Danielle Juteau, « La production de l'ethnicité ou la part réel de l'idéel ", Sociologie et sociétés 15, 2 (1983) : 39-54.

4 Miguel Simao Andrade, «La Commission des écoles catholiques de Montréal et l'intégration des immigrants et des minorités ethniques à l'école française de 1947 à 1977 ", Revue d'histoire de l'Amérique française 60, 4 (printemps 2007) : 455-486; Donat Taddeo et Raymond Taras, Le débat linguistique au Québec, la communauté italienne et la langue d'enseignement (Montréal : PUM, 1987), 246; Michel Laferrière, "L'éducation des enfants des groupes minoritaires au Québec : de la définition des problèmes par les groupes eux-mêmes à l'intervention de l'État ", Sociologie et sociétés 15, 2 (octobre 1983) : 117-131.

5 Soulignons cependant la contribution récente de Robert Gagnon, «Entre la foi et l'ouverture aux autres : la CECM, la promotion des valeurs chrétiennes et l'intégration des communautés culturelles, 1973-1998 " in Jean-Michel Lacroix et Paul-André Linteau, dir., Vers la construction d'une citoyenneté canadienne (Paris: Presses de la Sorbonne Nouvelle, 2006) : 77-86.

6 Pour une analyse comparative entre systèmes nationaux, voir Marie McAndrew, Immigration et diversité à l'école (Montréal : PUL, 2001), 263. Pour une synthèse générale, voir Marie McAndrew et Jean-Pierre Proulx, «Éducation et ethnicité au Québec : un portrait d'ensemble ", in Marie McAndrew et France Gagnon, dir., Relations ethniques et éducation dans les sociétés divisées (Paris: L'Harmattan, 2002), 85-109.

7 Fernand Harvey, "L’ouverture du Québec au multiculturalisme, 1900-1981 ", Études canadiennes/Canadian Studies, 21, T. 2 (1986) : 219-228; Julien Bauer, Les minorités au Québec (Montréal : Boréal Express, 1994), 60.

8 Rosalind Zinman, «Developments and Directions in Multicultural/Intercultural education, 1980-1990, The Province of Quebec ", Canadian Ethnic Studies 33, 2 (1991) : 65-80.

9 Marie McAndrew and Patricia Lamarre, «The Integration of Ethnic Students Fifteen Years After Bill 101 : Linguistic and Cultural Issues Confronting Quebec's French Language Schools ", Canadian Ethnic Studies 2, 2 (1996) : 40-63. 
10 Robert Gagnon, Histoire de la CECM (Montréal : Boréal, 1996), 298-307; Jocelyne Durand et al., La déconfessionnalisation de l'école ou le cas de Notre-Dame-des-Neiges (Montréal : Éditions Libre Expression, 1980), 275.

11 Nous avons systématiquement dépouillé et analysé le Livre des délibérations des commissaires qui contient les décisions, les recommandations et les politiques adoptées par la Commission. Parmi les autres documents consultés, mentionnons les études (rapports, enquêtes, statistiques) du Bureau de l'accueil (1972-1987) et de l'Office des relations interculturelles (1988- ) ainsi que les revues, bulletins d'informations, circulaires, journaux et publications internes de la CECM : Nouvelles (1976-1986), Compact (1976-1982), CECM (1982-1989), L'École montréalaise (1989- ), Dimensions (1981-1993) et Osmose (1991-1998).

12 Jocelyn Berthelot, Apprendre à vivre ensemble, Immigration, société et éducation (Montréal : CEQ, 1990), 25-26; Gouvernement du Québec, ministère des Communautés culturelles et de l'Immigration, Direction des études et de la recherche, Caractéristiques des immigrants admis au Québec 1981-1990, juin 1991, 39.

13 Danielle Juteau, "Du dualisme canadien au pluralisme québécois » in McAndrew et Gagnon, dir., Relations ethniques et éducation dans les sociétés divisées (Paris : l'Harmattan, 2000), 14-25; Denise Helly, "The Political Regulation of Cultural Plurality :

Foundations and Principles ", Canadian Ethnic Studies 25, 2 (1993) : 15-35; Fernand Harvey, "L'ouverture du Québec », 219-228.

14 Cité par Juteau, "Du dualisme canadien », 21.

15 En 1985, une étude des perceptions des leaders ethniques indique que $74 \%$ des répondants approuvent le PELO. Voir Michel Laferrière et al., "L'école et l'intégration des communautés ethno-culturelle au Québec : une étude des perceptions des leaders ethniques ", Éducation canadienne et internationale 14, 1 (1985), 102-103; McAndrew, Immigration et diversité , 49-75.

16 Conseil supérieur de l'éducation, L'éducation interculturelle (Québec : Éditeur officiel, 1983), 45; Ministère de l'Éducation du Québec, L’école québécoise et les communautés culturelles (Rapport Chancy) (Québec : Éditeur officiel, 1985), 180; Conseil supérieur de l'éducation, Les défis éducatifs de la pluralité (Québec : Éditeur officiel,1987), 43; Ministère de l'Éducation du Québec, L'école québécoise et les communautés culturelles (Rapport Latif) (Québec : Éditeur officiel, 1988), 99.

17 Marc-Yves Volcy, «La sensibilisation interculturelle du personnel scolaire par le ministère de l'Éducation du Québec : continuités et changements ", in Fernand Ouellet, dir., Les institutions face aux défis du pluralisme ethnoculturel (Québec: IQRC, 1995), 457-473; Marie McAndrew, «Le traitement du racisme, de l'immigration et de la réalité multi-ethnique dans les manuels scolaires francophones au Québec ", Canadian Ethnic Studies 18, 2 (1986) : 130-142.

18 McAndrew, Immigration et diversité, 112.

19 La CECM adopte sa politique d'éducation interculturelle en juin 1986 tandis que la Commission des écoles protestantes du Grand Montréal et la Commission scolaire Sainte-Croix font de même en juin et en octobre 1989 respectivement. Pour une analyse comparative voir Linda Pietrantonio, «De la gestion idéologique de la diversité ethnoculturelle : sens et limites» (mémoire de maîtrise, Université de Montréal, 1991), 216; Rosalind Zinman, "Developments and Directions », 65-80.

20 Parmi les écoles les plus multiethniques, mentionnons les écoles Lambert Closse (72,6 \%), Jean-Jacques Olier (73,6 \%), Saint-Jean Baptiste (80,9 \%), Saint-Simon Apôtre $(81,1 \%)$, Saint-Pascal Baylon (85,3\%), Saint-Léon (88,1\%), Les Enfants du Monde $(89,7 \%)$, N-D-de-la-Défense (90,4\%), Barthélémy-Vimont(92,8 \%) et Du NouveauMonde (100 \%). CECM, Rapport sur la population scolaire d'origine ethnique en 1985-1986, décembre 1986, 3, 29-33; CECM, Statistiques sur les origines ethniques des élèves de la CECM, la langue maternelle et la langue parlée à la maison, janvier 1973, 3, Archives de la Commission scolaire de Montréal, anciennement la CECM (ACSDM). 
21 Dans les écoles françaises de la CECM, les trois plus importantes communautés ethniques sont les Haïtiens (5 073), les Italiens (4 981) et les Portugais (2 510). Les quatre langues les plus parlées sont le créole (4 017), l'italien (3 071), l'espagnol (2 530) et le portugais (1 856). Voir Bureau de l'accueil et de l'admission, Rapport sur la population scolaire d'origine ethnique en 1985-1986, CECM, décembre 1986, 2, ACSDM.

22 Pierre Andreani et al, Étude comparative sur l'apprentissage du français entre les enfantsimmigrants en classe d'accueil et les enfants-immigrants en insertion directe, CECM, mai 1978, 119, ACSDM; Guy Pelletier et Manuel Crespo, Le jeune immigrant dans le système scolaire, une étude socio-scolaire réalisée sur les finissants des classes d'accueil de la CECM, CECM, septembre 1979, 125, ACSDM; Robert Attar, Le retard scolaire chez les jeunes immigrants, Bureau de l'accueil, 7 juin 1982, 17, ACSDM; Anne Laperrière, L'intégration socio-scolaire des enfants immigrants dans les écoles de milieux socioéconomiquement faibles : une recherche exploratoire (Montréal : Conseil scolaire de l'île de Montréal, 1983), 76.

23 Livre des délibérations des commissaires (LDC), 13 juillet 76, 10 mai 1977; Viateur Ravary, Rapport sur la situation des élèves haïtiens dans les écoles de la CECM, Service des études, 28 avril 1977, 13, ACSDM.

24 Yvan Dufour, Les élèves hä̈tiens à la CECM, Bureau du perfectionnement de l'enseignement, 14 décembre 1976, 2, ACSDM.

25 Jacques Charles-Pierre, "L'élève haïtien et l'école québécoise ", Québec français 53 (mars 1984) : 63-67; Michel Laferrière, «L'éducation des enfants ", 127.

26 Robert Attar, Rapport sur l'accueil et l'intégration des Haïtiens en milieu scolaire, Bureau de l'accueil, $1^{\text {er }}$ avril 1975, 1, ACSDM. Ne connaissant pas le pourcentage des élèves haïtiens chez les allophones de la CECM (le créole n'est pas employé comme catégorie dans les statistiques sur la population scolaire totale de 1975), il est impossible de déterminer leur poids relatif dans les classes d'accueil par rapport aux autres groupes linguistiques. Malgré cette limite évidente, les chiffres cités prouvent néanmoins que les classes d'accueil rejoignent au départ un très faible nombre de jeunes Haïtiens.

27 Centre haïtien d'orientation et d'information scolaire, Le jeune Haïtien en milieu scolaire montréalais (Montréal : Conseil scolaire de Montréal, novembre 1981), 5.

28 Précisons que la Commission protestante a déjà une longue expérience dans ses rapports avec la communauté noire qui s'explique par la présence nombreuse des Noirs anglophones dans ses écoles à partir de la fin des années 1960. Cette présence, source de tensions interethniques, amène la Commission à créer un poste d'agent de liaison en 1969. Voir Laferrière, "L'éducation des enfants ", 125-126.

29 En mars 1980, les enfants réfugiés d'origine vietnamienne représentent $39 \%$ des élèves de l'accueil. Le fait que la plupart d'entre eux ne soient pas scolarisés entraîne un alourdissement immédiat de la clientèle. Voir Bureau de l'accueil, Recherche sur le retard scolaire des jeunes immigrants du niveau secondaire, août 1980, 6; Robert Attar, Le retard scolaire, 17, ACSDM.

30 LDC, 16 juin 1982, ACSDM.

31 Coopération Nord-Sud en éducation, Le vécu scolaire interculturel dans les écoles de la CECM (Montréal : Coopération Nord-Sud, 1986), 22. Plusieurs études signalent l'accroissement des difficultés scolaires chez les allophones. Voir Mireille Ferland et Guy Rocher, La loi 101 et l'école primaire à clientèle pluriethnique, perception des intervenants (Québec : Éditeur officiel, 1987), 97; Conseil de la langue française, La place du français dans les écoles de langue française à clientèle pluriethnique de l'île de Montréal (Québec: Éditeur officiel, 1987), 34.

32 Plus de $75 \%$ des directeurs d'école et des enseignants-es rencontrent des difficultés de communication linguistique ou interculturelle lorsqu'ils entrent en contact avec les parents néo-québécois. Coopération Nord-Sud en éducation, idem. Pour un bref compte rendu du rapport, voir François Cadotte, "S'ouvrir aux autres cultures dans des écoles françaises ", L'Alliance 24, 2 (novembre-décembre 1986) : 39-41, ACSDM. 
33 Sur les rapports parents-école à la CECM, voir Marie McAndrew, "Relations entre l'école et les communautés culturelles " in Trait d'union: Actes du colloque sur les relations interculturelles (Montréal : CECM, 15 avril 1989) : 22-30, ACSDM.

34 Conseil de la langue française, L'école française à clientèle pluriethnique de l'île de Montréal (Québec : Éditeur officiel, 1987), 585; Robert Attar, Le changement démographique de la population scolaire des écoles françaises de la CECM et ses effets, Bureau de l'accueil, 29 juin 1983, 7, ACSDM; Pierre Noël, Rapport sur la problématique des tensions raciales et du racisme dans le milieu scolaire, Bureau de l'accueil, août 1984, 26, ACSDM.

35 Henry Égretaud, "Louis-Joseph Papineau : 25 ethnies, une semaine », L'Alliance 19, 6 (juin 1982) : 26-27; "Les immigrants dans nos écoles " L'Alliance 20, 3 (novembredécembre 1982) : 27-28.

36 LDC, 29 juin 1983, ACSDM; "Face à la montée du multiculturalisme : prévenir le racisme dans les écoles ", CECM, 2, 3 (15 octobre 1983), 2, ACSDM.

37 Robert Attar, Rapport d'étape du Comité consultatif pour le développement de l'éducation interculturelle, Bureau de l'accueil, 7 décembre 1982, 5; LDC, 18 janvier 1984, ACSDM.

38 En plus des insultes proférées par des élèves (" maudits nègres ", "sale noir ", etc), mentionnons les propos du personnel scolaire qui sont tout autant teintés de racisme primaire. Par exemple, le jour de son accueil à l'école, une élève noire s'est fait dire par son orienteur : "Tu fais vraiment pitié comme ceux de ta race ». Des élèves noirs qui parlaient créole dans leur classe se sont fait dire par leur enseignant : "Allez parler votre langue de sauvages ailleurs ». Voir Noël, Rapport sur la problématique, 18-23.

39 LDC, 20 novembre 1984, ACSDM.

40 Ces mesures sont généralement couronnées de succès. En 1986, l'agent de liaison Pierre Noël et le directeur de l'école Henri-Bourassa constatent une nette amélioration des relations interethniques et une régression des gestes racistes. Ainsi, on compte six parents haïtiens dans le comité d'école alors qu'il n'y en avait aucun en 1984. Voir Robert Cadotte, "Les tensions raciales diminuent ", L'Alliance 24, 1 (septembre-octobre 1986), 31-33; Robert Attar, Rapport sur les tensions raciales dans les écoles du secteur français de la CECM, Bureau de l'accueil, 24 septembre 1984, 10, ACSDM.

41 LDC, 11 avril et 20 novembre 1984; CECM, Politique de services aux élèves des communautés culturelles fréquentant les écoles françaises de la CECM, avril 1984, 18, ACSDM.

42 Rapport Chancy, 155.

43 LDC, 25 juin 1986, ACSDM.

44 Stéphane Crespo et Gisèle Painchaud, "Recueil de résumés de texte (1977-1991) ", in Le programme d'enseignement des langues d'origines (Montréal : Les publications de la faculté des sciences de l'éducation, Université de Montréal, 1993), 33-36.

45 CECM, Actes du colloque sur le Programme d'enseignement des langues d'origine : 10 ans après, 2 mai 1987, 54; Marie McAndrew et Simone Gress-Azam, Évaluation des services offerts dans le cadre du PELO et de son impact sur les élèves et sur les écoles, Bureau de l'accueil, 1987, 17, ACSDM.

46 Ferland et Rocher, La loi 101, 57-67.

47 Ministère de l'Éducation, L'ouverture du PELO à l'ensemble de la clientèle scolaire : Rapport final (Québec : Éditeur officiel du Québec, 1988), 42.

48 Simone Gress-Azzam, " PELO maintenant au secondaire ", L'école montréalaise, 2, 14, 1, ACSDM; Jacqueline Romano-Toramanian, «Le PELO, une mesure si mal aimée, si peu comprise ", L'école montréalaise, 8, 7 (16 décembre 1996), 7-8, ACSDM; McAndrew, Immigration et diversité, 50-75.

49 LDC, 30 septembre 1987; 27 avril et 14 septembre 1988; 18 octobre 1989, ACSDM.

50 Voir André Beauchesne, Éducation et pédagogie interculturelles : l'approche de formation (premier livret) (Montréal : Éditions du CRP, Faculté d'éducation, Université de Sherbrooke et CECM, 1991), 51-55. 
51 L'Alliance est un syndicat qui regroupe les enseignants et enseignantes de Montréal œuvrant aux niveaux primaire et secondaire.

52 Voir par exemple "L'intégration des immigrants ", Dimensions 2, 3 (avril 1981), 7-15; "La réalité multiethnique des écoles ", Dimensions 11, 1 (novembre 1989), 7-16; "Les immigrants dans nos écoles ", L'Alliance 23, 2 (novembre-décembre 1985), 21-32; Osmose 1, 1 (décembre 1990), ACSDM.

53 LDC, 8 février 1989; L'école montréalaise 1, 7 (2 avril 1990), ACSDM; Berthelot, Apprendre à viure, 47.

54 L'école montréalaise 2, 15 ( $1^{\mathrm{er}}$ avril 1991), 5, ACSDM.

55 Dans les années 1970, la CECM perd 100000 élèves. Entre 1976 et 1983, elle est obligée de fermer plus de 125 écoles, soit près du tiers. Cette décroissance se traduit par des coupures massives dans le personnel. Entre 1972 et 1981, le personnel scolaire (enseignants, cadres, professionnels, etc) a chuté de $25 \%$. Voir Robert Gagnon, Histoire de la CECM, 292-295.

56 CECM, Trait d'union, actes du colloque sur les relations interculturelles, avril 1989, 18-21; LDC, 12 avril 1989, ACSDM.

57 Jean-Pierre Proulx, «La CECM voudrait savoir si les parents veulent une école pour Québécois pure laine ", Le Devoir, 7 novembre 1989, 3; Cauchon, P. " Dénonciation unanime du sondage de la CECM ", Le Devoir, 10 novembre 1989, 3; André Pratte, "La CEPGM abandonne son projet d'école pour les enfants d'origine haïtienne ", $L a$ Presse, 18 janvier 1990, A3.

58 La ligue des droits et libertés, l'Institut Interculturel de Montréal, le Congrès Juif canadien, le Conseil National Indo-Canadien, le Centre communautaire sud-asiatique et la Maison d'Haïti ont également dénoncé la position du RSC. Patrick Grandjean et Marie-France Léger, "Michel Pallascio soulève indignation et critiques », La Presse, 15 novembre 1990, B4.

59 CECM, Office des relations interculturelles, Le développement et la promotion des relations interculturelles et interethniques à la CECM, Plan d'action, juin 1990, 52; LDC, 15 avril et 2 septembre 1992, ACSDM.

60 Pour une synthèse du débat opposant les tenants de l'éducation interculturelle et de l'éducation à la citoyenneté voir Fernand Ouellet, «Éducation interculturelle et formation interculturelle. Éléments de problématique ", Études ethniques au Canada 29, 2 (1997) : 32-57; McAndrew, Immigration et diversité, 147-175.

61 Pierre-Michel Laguerre et al., Pour mieux vivre ensemble dans une culture publique commune, CECM, Service des relations interculturelles, 1994, 66; Pierre-Michel Laguerre, Léducation interculturelle, où en sommes-nous?, CECM : Service des relations interculturelles, 1995, 63, ACSDM.

62 Robert Gagnon défend aussi cette thèse dans un récent article. Voir Robert Gagnon, «Entre la foi et l'ouverture aux autres : la CECM, la promotion des valeurs chrétiennes et l'intégration des communautés culturelles, 1973-1998 " in Jean-Michel Lacroix et Paul-André Linteau, dir., Vers la construction d'une citoyenneté canadienne, (Paris : Presses de la Sorbonne Nouvelle, 2006), 77-86.

63 Gagnon, Histoire de la CECM, 276.

64 Par exemple, le rapport Parent recommande, en 1966, l'établissement d'écoles non confessionnelles et, l'année suivante, le Comité catholique stipule que l'école catholique doit permettre l'exemption religieuse dans les écoles confessionnelles et établir un cours d'enseignement moral pour tous les élèves des parents qui en font la demande. Conseil supérieur de l'éducation, Règlements du Comité catholique du Conseil supérieur de l'éducation, Québec, juin 1967, ACSDM. Rapport Parent, "Diversité religieuse et culturelle et unité administrative ", $3^{\mathrm{e}}$ partie (Québec : Éditeur officiel, 1966).

65 Contrairement à l'école catholique où la foi imprègne l'ensemble de l'institution, depuis l'enseignement religieux jusqu'à la direction, en passant par la philosophie et le projet éducatif, l'école protestante est, à toutes fins pratiques, neutre et son projet éducatif 
heurte moins la liberté de conscience des groupes religieux minoritaires. L'enseignement religieux y est optionnel et diversifié et dans les écoles où la clientèle est majoritairement juive ou orthodoxe, la confessionnalité protestante ne représente plus qu'une façade juridique. Roderick MacLeod et Amy Ann Poutanen, A Meeting of the People : School Boards and Protestant Communities in Quebec, 1801-1998 (Montreal: McGill-Queen's, 2004), 472; Roger Magnuson, The Two Worlds of Quebec Education during the Traditional Era, 1760-1940 (London: The Althouse Press, 2005), 267.

66 Jean Perras, L'exemption du cours d'enseignement religieux ou le cours de sciences morales au secondaire, décembre 1974, 9, ACSDM.

67 Énoncé de principe de l'Association québécoise pour l'application du droit à l'exemption religieuse, 27 mars 1976, 2; Henri Van Oorshot, "La liberté religieuse existe-t-elle à la CECM? ", Compact 1, 3 (décembre 1976), 2; LDC, 30 juin et 21 octobre 1976; 22 mars 1979; 6 mars 1985, ACSDM.

68 Le MSC, fondé en 1973 par l'Association des parents caholiques, fait élire 18 des 19 candidats en lice. Le faible taux de participation aux élections scolaires conjugué à l'organisation efficace du MSC explique, en grande partie, comment la minorité ultra catholique a réussi à remporter et à conserver le pouvoir.

69 Louis Bouchard, ancien président de l'Association des parents catholiques, commissaires de la CECM de 1973 à 1987 et principal penseur du MSC, exerce une influence déterminante sur la position idéologique de la CECM au sujet de la confessionnalité et de la place de la religion à l'école. Voir Gagnon, Histoire de la CECM, 285-291.

70 LDC, 12 janvier 1978, ACSDM. En 1977, les statistiques concernant les croyances religieuses des élèves fréquentant les classes d'accueil se répartissent ainsi : catholiques $(65 \%)$, orthodoxes $(11 \%)$, musulmans $(10,5 \%)$, protestants $(8,5 \%)$ et autres $(5$ $\%)$. Robert Attar, L'enseignement religieux en classes d'accueil, Bureau de l'accueil, 23 septembre 1977, 3, ACSDM.

71 Voir Gagnon, Histoire de la CECM, 300-301.

72 Jocelyne Durand et al., La déconfessionnalisation de l'école, 275; Anita Caron, Les parents et le statut confessionnel au Québec (Montréal : PUQ, 1984), 206.

73 Rappelons que la loi du gouvernement du Québec adoptée en juin 1964 donne pouvoir au comité catholique de reconnaître et de révoquer le statut confessionnel des écoles. En 1974, le comité catholique avait déclaré officiellement catholiques toutes les écoles relevant de la CECM, y compris l'école NDDN. François Hamel, Les enjeux de la confessionnalité au Québec (Montréal : Les Presses d'Amérique, 1995), 66-67.

74 Cité dans Jocelyne Durand et al, op.cit., 166; LDC, 21 février 1980, ACSDM.

75 En 1984-1985, les commissions scolaires catholique et protestante de Montréal s'unissent pour faire avorter le projet de loi 3 visant à remplacer les commissions scolaires confessionnelles par des commissions scolaires linguistiques. François Hamel, Les enjeux de la confessionnalité, 65-84; Conseil supérieur de l'éducation, Pour une évolution équitable des structures scolaires du Québec, Avis au ministre de l'Education, Québec : janvier 1986, 4, ACSDM.

76 LDC, 3 novembre et 11 décembre 1985, ACSDM; « École et religions », L'Alliance 23, 5 (mai-juin 1986), 19-37.

77 Pour expliquer la forte présence des minorités à la Commission protestante, il faut bien sûr rappeler qu'avant l'adoption de la loi 101, en 1977, la quasi-majorité d'entre elles choisissaient l'école anglo-protestante pour des raisons de mobilité socio-économique. De plus, la Commission protestante avait tout intérêt à favoriser l'intégration des minorités à la communauté anglophone afin de conserver son poids démographique et politique. Pourtant, le fait que les minorités continuent d'opter massivement pour l'école franco-protestante après 1977 plutôt que l'école franco-catholique prouve que l'attrait de la langue anglaise n'explique pas tout et que le facteur religieux est loin d'être secondaire dans ce choix. Jean-Pierre Proulx, « Le pluralisme religieux : les voix de 
l'impasse ", in André Charron, dir., École et religion, le débat, (Québec : Fides, 1995), 169-170.

78 Ghislaine R.-Godbout, «S'accommoder n'est pas commode », Osmose 2, 4 (mai 1992), 1, ACSDM.

79 Cette norme juridique, issue du droit du travail, date d'un jugement de la Cour suprême du Canada, en 1985, sur le cas d'une plainte de discrimination religieuse par une femme adventiste, employée de Sears. La Cour juge que dans le cas d'une discrimination, l'institution ou l'entreprise ont l'obligation de s'entendre avec le plaignant, à moins que cela ne cause une contrainte excessive (limite des ressources, atteinte au droit du public, bon fonctionnement de l'institution). Les motifs de discrimination sont divers : âge, sexe, grossesse, handicap, religion.

80 Le Conseil des Communautés culturelles et de l'Immigration est, à notre connaissance, le premier organisme québécois à se pencher sur la question, mais sa contribution n’a aucune valeur légale. Voir Conseil des Communautés culturelles et de l'Immigration, Gérer la diversité dans un Québec francophone, démocratique et pluraliste : Principes de fond et de procédure pour guider la recherche d'accommodements raisonnables (Montréal : CCCI, 1993), 103.

81 Lise Labelle, "Témoignages », Osmose 2, 4 (mai 1992), 2, ACSDM.

82 McAndrew, Immigration et diversité, 134-140.

83 Les termes " pompier de l'interculturel " et " Monsieur Accommodement Raisonnable " que les journalistes accolleront ultérieurement à M. Bergman Fleury, conseiller interculturel de la CECM, sont peut-être anecdotiques, mais ils illustrent bien la nature particulière des rapports sociaux entre l'école et les minorités. Ces rapports évoluent au gré des circonstances et se déroulent sur le terrain entre des individus qui acceptent de jouer le jeu de la négociation et du dialogue sans avoir recours au système judiciaire. Par exemple, en 1998, soit quatre avant la cause judiciaire du kirpan à la Commission scolaire Marguerite-Bourgeoys, la polyvalente Lucien-Pagé de la Commission scolaire de Montréal et $\mathrm{M}$. Fleury arrivent à une entente négociée avec un jeune sikh sans avoir à recourir aux tribunaux. Après de longues recontres de discussions, le jeune accepta de porter, au cou, une chaîne avec un kirpan symbolique. Voir Katia Gagnon, "Au-delà du Kirpan ", La Presse, 29 mai 2007; Katia Gagnon, "Monsieur Accommodement Raisonnable ", La Presse, 29 mai 2006.

84 Le rapport propose une laïcité ouverte permettant l'enseignement culturel des religions. Il faut toutefois attendre 2007 pour que le gouvernement québécois décide de remplacer l'enseignement confessionnel religieux par un programme «Éthique et culture des religions ". Rapport du Groupe de travail sur la place de la religion à l'école, Laïcité et religions. Perspectives nouvelles pour l'école québécoise. Voir le document en ligne http:// www.meq.gouv.qc.ca/REFORME/religion/inter.htm

85 Une simple recherche sur Internet suffit pour constater l'ampleur du débat dans l'espace médiatique et politique québécois. Cherchant à calmer la grogne de l'opinion publique, mais surtout à éclairer un débat extrêmement complexe, le gouvernement libéral de Jean Charest met sur pied, le 8 février 2007, une Commission d'enquête sur les pratiques d'accommodements reliées aux différences culturelles, présidé par deux universitaires, le philosophe Charles Taylor et l'historien Gérard Bouchard.

86 La CSDM est la première institution scolaire à inscrire le concept "d'accommodement raisonnable réciproque " dans sa politique d'éducation interculturelle. Aussi, prendelle soin de définir clairement ce terme : il s'agit d'un « arrangement particulier 1 - fondé juridiquement sur les chartes canadienne et québécoise des droits et libertés, 2- caractérisé par une exception au respect d'une règle en vigueur afin de cesser une discrimination (qui, n'étant pas "illicite et intentionnelle" est causée par cette règle et interdite par ces chartes), 3-résultant d'une recherche de solution négociée entre les parties concernées, sans contrainte excessive pour son application et 4-respectant 
"l’ordre public et le bien-être général" de la collectivité ". Voir http://www.csdm.qc.ca/ Csdm/Administration/pdf/POLITIQUE_INTERCULTURELLE_21_juin_06.pdf Un sondage mené récemment par la CSDM auprès de ses écoles montre que cette approche fonctionne bien. En 2006, 145 écoles ont reçu 894 demandes d'accommodements et sur ces demandes, 659 ont été acceptées, soit $77 \%$. Seulement $0,3 \%$ des élèves ont demandé des accommodements pour des motifs religieux et aucun cas de refus ne s'est traduit par une plainte à la Commission des droits de la personne. Voir Lia Lévesque, "La CSDM demande de légiférer sur certains accommodements ", La Presse, 27 novembre 2007.

87 Tommy Chouinard, "Les écoles démunies face aux demandes religieuses ", La Presse, 21 septembre 2007. 